Published in final edited form as:

J Theor Biol. 2007 February 7; 244(3): 451-462.

\title{
A Dynamic Model for Induced Reactivation of Latent Virus
}

\author{
G.M. Keplere,a, H.K. Nguyen ${ }^{\mathrm{e}, \mathrm{b}}$, J. Webster-Cyriaque ${ }^{f, c}$, and H.T. Banks ${ }^{\mathrm{e}, \mathrm{d}}$ \\ e Center for Research in Scientific Computation, North Carolina State University, Raleigh, N.C. 27695-8205, \\ USA \\ f Department of Dental Ecology, School of Dentistry, University of North Carolina, Chapel Hill, N.C., USA
}

\begin{abstract}
We develop a deterministic mathematical model to describe reactivation of latent virus by chemical inducers. This model is applied to the reactivation of latent KSHV in BCBL-1 cell cultures with butyrate as the inducing agent. Parameters for the model are first estimated from known properties of the exponentially growing, uninduced cell cultures. Additional parameters that are necessary to describe induction are determined from fits to experimental data from the literature. Our initial model provides good agreement with two independent sets of experimental data, but also points to the need for a new class of experiments which are required for further understanding of the underlying mechanisms.
\end{abstract}

\section{Keywords}

Reactivation of Latent Virus; Mathematical Model; KSHV; Herpes Virus

\section{Introduction}

\begin{abstract}
Many viral pathogens establish latency and are dormant. The presence of inducers leads these pathogens to reactivate and replicate, aiding their transmission and contributing to disease development. In addition, there is increasing evidence in the literature for the importance of polymicrobial infections in which microorganisms interact in a synergistic fashion, impacting both pathogenesis and maintenance of health. Among these, virus-bacteria interactions have been described, including reactivation of latent virus by metabolic end products of anaerobic bacteria. A shift in the balance of the flora often controlled by the intact immune system may reflect significant morbidity particularly in the immune suppressed host. The relationships between viral pathogens and their inducing agents have not previously been described mathematically. Therefore, to begin to understand quantitative relationships between pathogens and their inducing agents, particularly in a polymicrobial environment, we have developed a preliminary mathematical model that describes the reactivation of latent herpes virus by an inducer that behaves similarly to the metabolic end products of anaerobic bacteria.
\end{abstract}

Send correspondence and reprint requests to: G.M. Kepler, Center For Research in Scientific Computation, Box 8205 North Carolina State University, Raleigh, NC 27695-8205, USA, Telephone, +1 919-515-8966; Fax, +1 919-515-8967

a email: gmkepler@ncsu.edu

bemail: hknguyen@ncsu.edu

cemail: cyriaquj@dentistry.unc.edu

demail: htbanks@ncsu.edu

Publisher's Disclaimer: This is a PDF file of an unedited manuscript that has been accepted for publication. As a service to our customers we are providing this early version of the manuscript. The manuscript will undergo copyediting, typesetting, and review of the resulting proof before it is published in its final citable form. Please note that during the production process errors may be discovered which could affect the content, and all legal disclaimers that apply to the journal pertain. 
Currently, there are eight known herpes viruses that infect humans. After primary infection, the virus remains latent in specific types of host cells that may be different from the types of cells targeted for primary infection. Latent virus persists in the cell nucleus as episomal DNA until it is reactivated, beginning a program of lytic replication and lysis, and leading to a new (sometimes asymptomatic) round of infection and latency. The lytic replication program is characterized by a temporal cascade of gene expression that is typically grouped into three phases: Immediate Early, Early, and Late. During Immediate Early and Early phases infected cells produce viral proteins that are necessary for viral DNA synthesis, which occurs at the end of the Early phase. During the Late phase of the reproductive cycle the host cell is directed to make the structural proteins necessary for viral packaging.

The exact mechanisms by which latent virus becomes reactivated and begins lytic replication are not entirely known. However, it has been established that inducing agents such as Tetradecanoyl Phorbol Acetate (TPA), sodium butyrate, and other short chain fatty acids (SFAs) can induce lytic replication of Kaposi's Sarcoma-associated Herpes virus (KSHV) and Epstein-Barr virus (EBV) $[11,36,37]$. In addition, recent experiments have shown that the spent media from gram negative bacteria cultures, such as P. Gingivalis and P. Intermedia, which contains SFAs (e.g., iso-valeric, n-butyric acid, and propionic acid), can also induce latent KSHV to begin lytic replication [28].

A mechanism for bacterial reactivation of latently infected cells has strong health implications for the oral environment as well as the gut and GI tract, where there may be large numbers of gram negative bacteria in the presence of latently-infected cells. Reactivation of latent herpes viruses are a major health concern for immune-compromised individuals, such as those with AIDS. Understanding the role of anaerobic bacteria in reactivation of latent herpes viruses may have important health consequences if there is similar reactivation of latent episomal HIV by bacterial metabolic end products.

To our knowledge there has been no mathematical modeling treatment of viral reactivation at the cellular level. Much of the mathematical modeling of herpes viruses has focused, understandably, on modeling at the epidemiological level (e.g., [7,16]). Recently, Wang et al., used HHV-6 infection as a stimulus for studying cellular changes in the T cell immune system under pathological conditions [33]. Their study included data from the literature, clinical data, and cell culture data. Their model agreed well with data, but focused on the viral load and T cell response. Clearly there is a need and opportunity to understand viral latency and reactivation, especially since this is a characteristic feature of herpes virus infection.

In this manuscript we report on a first deterministic mathematical model that we have developed to describe reactivation of latent virus by chemical inducers. In particular, we develop this model in the context of the reactivation of latent KSHV in BCBL-1 (body-cavity-based lymphomas-1) cells with butyrate as the inducing agent. KSHV, also known as Human Herpesvirus-8 (HHV-8), is a gamma herpes virus that is responsible for Kaposi's Sarcoma tumor development and other lymphoproliferative disorders such as Castleman's disease and primary effusion lymphoma. KSHV latently infects epithelial and lymphoid cells that are present in the oral environment and reactivation of latent virus in B cells may play a role in the pathogenesis of Kaposi's sarcoma [25]. BCBL-1 cells are an immortalized cell line derived from body-cavity-based lymphomas that are latently infected with multiple copies of KSHV, but not Epstein-Barr virus (EBV) [27]. Latently infected B-cell lines (such as BCBL-1) are considered to be major tools for studying KSHV biology [13], as is reflected by the considerable amount of experimental literature utilizing these cell lines. We model butyrate as the motivating inducing agent because it is commonly used in experiments to induce reactivation of latent viruses in the particular cell system that we are modeling (BCBL-1 cells), and therefore there is greater availability of published experimental data, and because, as an SFA, butyrate behaves 
in a manner similar to the metabolic end products of gram negative bacteria. In subsequent stages we intend to use our own experimental data (JW-C) to model KSHV reactivation that is induced by the metabolic end products of gram negative bacteria.

We briefly outline our contributions in this paper. In Section 2 we discuss compartments we believe necessary in any first attempt to model reactivation of latent viruses. The three major compartments, depicted schematically in Fig. 1, are host cells with lytic virus, host cells with latent virus, and host cells that have become nonviable through death from several causes. These compartments are a basis for the dynamics of cell populations as modeled in Section 3 under the assumptions that no induction is present, although there is a low level of ongoing spontaneous reactivation. This first step, based on our approximations to what is currently understood through experiments to date, is necessary and employs a standard approach using mass balance in compartmental systems. To our knowledge, this fundamental step has not been previously carried out. But because we are ultimately interested in amounts of virus (latent and replicating) available (these are the quantities we expect to be able to measure), we couple these mass balance laws for host cells (host cell dynamics) with dynamics for the number of copies of virus (viral dynamics). This results in the coupled system (3) for uninduced dynamics of host cells and viruses. In Section 4 we modify the coupled model (3) for uninduced cell/ viral dynamics to now include induction of latent virus by a generic inducing agent $s$, using the actions of butyrate as a typical agent for modeling purposes.

In Section 5 we estimate parameters for the uninduced model from biological considerations (based on average behavior and known properties, i.e., "book values" of uninduced in vitro cell cultures) and under the tacit assumptions that these parameters will be independent of the presence of inducers. In Section 6 we turn to model simulations. We first use two independent data sets [36,37] from different laboratory groups along with literature based experimental considerations to fix certain parameter values. We then carry out simulations for the uninduced models to compare longitudinal limits (i.e., asymptotic behavior) and equilibria with the data of [36,37]. These equilibrium states are used as initial values for the numerical simulations where inducing agents are introduced. Before these simulations can be performed we must estimate induction rate functions which cannot be found in the literature. For our preliminary model we chose parameterized affine functions for the induction rates and used an Ordinary Least Squares (OLS) formulation with the data of $[36,37]$ to estimate the resulting parameters in the affine functions. The reasonably sophisticated mathematical and statistical methodology used to estimate the parameters and associated confidence intervals is detailed in the Appendix. In the Discussion and Conclusion we use the modeling considerations and results to suggest that new experiments are needed for further validation of our current and future generation models.

The model presented here establishes a general framework for modeling the effect of other inducing agents that act through histone-deacetylase (HDAC) inhibition, including other SFA's produced by the metabolic processes of gram negative bacteria. As such, it may also be applied to other latent virus systems that are induced to replicate via HDAC inhibition, such as EBV, HIV, and HCMV.

But before proceeding with the modeling effort, we first discuss why we are making this effort and what we believe our contributions are. We first note that wide use of mathematical modeling to aid in the understanding of scientific hypotheses is found and historically accepted in the engineering community. More recently, investigators in the biosciences have begun to recognize the value of mathematical modeling coupled with experimental investigations to enhance understanding of mechanisms, pathways, anomalies, etc. The modeling process itself is (or should be) most often an iterative process. As depicted schematically in Fig. 2, one can distinguish in it a number of rather separate steps which usually must be repeated. One begins 
with the real system under investigation and pursues the following sequence of steps: (i) empirical observations, experiments, and data collection; (ii) formalization of properties, relationships and mechanisms which result in a biological or physical model; (iii) abstraction or mathematization resulting in a mathematical model; (iv) formalization of uncertainty/ variablity in model and data resulting in a statistical model; (v) model analysis, interpretation and comparison (with the real system) of the conclusions, predictions, and conjectures obtained from step (iv); (vi) changes in "understanding" of mechanisms, pathways, etc., in the real system; and (vii) design of new experiments.

Our own efforts reported on in this paper must be considered a first step in the iterative modeling process and grew out of our desire to develop quantitative models related to the experimental efforts in the laboratories of one of the authors (JC-W). A major expected outcome of our collaborations is suggestion of needed experiments. Moreover, we anticipate that these efforts will guide and inform the design of new in vitro and in vivo experiments.

\section{Modeling Compartments}

We describe the dynamics of the host cells and viral DNA copies using a set of ordinary differential equations (ODEs). A schematic diagram of the ODE model compartments is shown in Fig. 1. Latent $L$ and lytic $R$ copies of viral DNA reside in the nuclei of host cells. We make the following "all or nothing" simplifying assumption: within a given host cell, nuclear viral DNA copies are either all latent or all in a lytic replication program. Therefore, there are two types of host cells, host cells $H_{R}$ with lytic virus only or host cells $H_{L}$ with latent virus only. Host cells that die are added to a nonviable host cell compartment $N$.

In future work, we plan to superimpose a probability distribution on the parameters to better approximate mixed conditions where a host cell may contain both latent and lytic virus in varying levels. Such a modeling technique was successfully used in cellular level HIV models to account for variable length (with uncertainty) pathways in [3]. In models of this type the state variables are the expected values of concentrations (or of numbers of cells) resulting in delay differential equation models embodying uncertainty. We do not pursue this level of refinement in the initial model developed here.

During the Early Phase of the lytic program, exponential-like replication of viral DNA $R$ takes place in replication compartments [34], where the progeny of replicating virus become replication templates themselves [14]. The intracellular viral DNA compartment $V_{I}$ represents viral DNA copies that are no longer targets for replication and are available for encapsulation. After envelopment, the virus is released as free virions $V_{F}$. We assume that free virions that are produced are not capable of reinfecting the host cells [5,21].

\section{Mathematical Model for the Uninduced Case}

\section{Host cell dynamics}

Latently infected host cells $H_{L}$ replicate and die (natural death) with rate constants $\beta_{L}$ and $d_{L}$, where $\beta_{L}>d_{L}$. As part of the lytic cycle, herpes viruses block the cell cycle in $\mathrm{G}_{0} / \mathrm{G}_{1}$ and block cell-initiated apoptosis $[15,18,35]$. Therefore, we assume that $\beta_{R}=0$ and $d_{R}=0$ for lytic host cells $H_{R}$. There is an additional mechanism for cell death due to the production of virus and the resulting cell lysis [29]. We model this viral-induced death rate as a function of the average amount of intracellular virus that accumulates $d_{I}\left(V_{I}\right) H_{R}$, where $V_{I}=V_{I} / H_{R}$. Nonviable cells are in a process of disintegration into smaller fragments and leave the $N$ compartment with a rate $\mu N$. 
Experimental observations of uninduced cell cultures indicate that some fraction of host cells will, through spontaneous reactivation, have lytic virus $[8,22,26]$. Therefore, we include terms for spontaneous reactivation of latently infected cells with a rate constant $\alpha_{0}$. We have previously shown that a corresponding deactivation rate constant $\rho$ must be zero or the number of latent copies per host cell $H_{L}$ will be drifting with time [21].

With these considerations in mind, we model the host cell dynamics in the uninduced case by

$$
\begin{aligned}
& \frac{d H_{L}}{d t}=\left(\gamma_{L}-a_{0}\right) H_{L} \\
& \frac{d H_{R}}{d t}=a_{0} H_{L}-d_{I}\left(\nabla_{I}\right) H_{R} \\
& \frac{d N}{d t}=d_{L} H_{L}+d_{I}\left(\nabla_{I}\right) H_{R}-\mu N,
\end{aligned}
$$

where $\gamma_{L}=\beta_{L}-d_{L}$

\section{Viral dynamics}

Each time a BCBL-1 host cell replicates the latent viral DNA copies are also replicated in the daughter cell, producing, on average, $L$ copies of latent virus. In addition, each dying latent host cell destroys $L$ copies of latent virus $L$. Similarly, each dying lytic host cell destroys, on average, $R$ and $V_{I A}$ lytic and intracellular copies of virus. The reactivation of latent and lytic virus follows in a manner similar to the terms above. Therefore, the uninduced model for the viral DNA dynamics is given by the set of equations

$$
\begin{aligned}
& \frac{d L}{d t}=\left(\gamma_{L}-a_{0}\right) H_{L} I \\
& \frac{d R}{d t}=a_{0} I H_{L}-d_{I}\left(\nabla_{I}\right) H_{R} R+(\kappa-q) R \\
& \frac{d V_{I}}{d t}=q R-p V_{I}-d_{I}\left(\nabla_{I}\right) H_{R} V_{I} \\
& \frac{d V_{F}}{d t}=p V_{I}
\end{aligned}
$$

where $\kappa R$ is the replication rate for lytic viral DNA, $q R$ is the rate at which lytic virus moves to the intracellular compartment, and $p V_{I}$ is the rate at which the intracellular DNA is packaged and excreted as virions $V_{F}$.

\section{Uninduced cell and viral dynamics}

Using $L=L / H_{L}, R=R / H_{R}$ and $V_{I}=V_{I} / H_{R}$, we can write the full uninduced model a

$$
\begin{aligned}
& \frac{d H_{L}}{d t}=\left(\gamma_{L}-a_{0}\right) H_{L} \\
& \frac{d H_{R}}{d t}=a_{0} H_{L}-d_{I}\left(\nabla_{I}\right) H_{R} \\
& \frac{d N}{d t}=d_{L} H_{L}+d_{I}\left(\nabla_{I}\right) H_{R}-\mu N \\
& \frac{d L}{d t}=\left(\gamma_{L}-a_{0}\right) L \\
& \frac{d R}{d t}=a_{0} L-\left(d_{I}\left(\nabla_{I}\right)-\kappa+q\right) R \\
& \frac{d V_{I}}{d t}=q R-\left(p+d_{I}\left(\nabla_{I}\right)\right) V_{I} .
\end{aligned}
$$


The solution for $V_{F}$ is easily obtained from the above solutions and can be written as

$$
V_{F}(t)=V_{F 0}+\int_{t_{0}}^{t} p V_{I}(u) d u
$$

\section{Mathematical Model for the Induced Case}

We next modify the uninduced model (3) to include the actions of an inducing agent $s$. The main affect of the inducing agent is to increase the rate at which latent virus becomes reactivated $\left(\alpha(s) \geq \alpha_{0}\right)$. In addition, inducing agents such as butyrate and valproate may also cause host cell death $\left(\delta_{L}(s)\right.$ and $\left.\delta_{R}(s)\right)$ through activation of host cell genes [6,20,36]. We make a simplifying assumption that inducing mechanisms that would initiate cell apoptosis would also lead to viral reactivation and, therefore, assume that $\delta_{L}=0$. With these additional terms the equations for the induced case become

$$
\begin{aligned}
& \frac{d H_{L}}{d t}=\left(\gamma_{L}-a(s)\right) H_{L} \\
& \frac{d H_{R}}{d t}=a(s) H_{L}-\left(d_{I}\left(\nabla_{I}\right)+\delta_{R}(s)\right) H_{R} \\
& \frac{d N}{d t}=d_{L} H_{L}+\left(d_{I}\left(\nabla_{I}\right)+\delta_{R}(s)\right) H_{R}-\mu N \\
& \frac{d L}{d t}=\left(\gamma_{L}-a(s)\right) L_{I} \\
& \frac{d R}{d t}=a(s) L-\left(d_{I}\left(\nabla_{I}\right)+\delta_{R}(s)-\kappa+q\right) R \\
& \frac{d V_{I}}{d t}=q R-\left(p+d_{I}\left(\nabla_{I}\right)+\delta_{R}(s)\right) V_{I}
\end{aligned}
$$

and

$$
V_{F}(t)=V_{F 0}+\int_{t_{0}}^{t} p V_{I}(u) d u
$$

\section{Parameter Estimation}

We estimate that the rate at which lytic virus moves from the $R$ compartment to the $V_{I}$ compartments is inversely proportional to the time that the lytic virus spends in the lytic program of gene expression and replication $q=1 / T$, where $T$ is the approximate time it takes to complete the lytic program (approximately 48 hours). For the sake of simplicity we model the viral-induced death rate as a simple affine function $d_{I}\left(V_{I}\right)=c V_{I}$.

We argue that it is reasonable to assume that on average the number of latent copies of viral DNA per latently infected host cell is constant $L / H_{L}=n$ for the following reasons. Although it has been shown in vivo that the viral-genome copy number varies over three orders of magnitude in neurons latently infected with herpes simplex virus [30], we are modeling a cell culture system, essentially established from a single immortalized cell and not an in vivo collection of cells. Each time a BCBL-1 host cell replicates the latent viral DNA copies are also replicated in the daughter cell. In addition, this is a well-established cell line that has been and continues to be used for many experiments related to KSHV latency and reactivation and it would be evident if this basic property of the cells was drifting in time.

Experimentalists typically observe the following quantities: fraction of lytic host cells $a_{s}$, fraction of nonviable host cells $N_{r}$, host cell doubling time $D_{p}$, and average copies of viral DNA per cell $n_{T}$ (viral load), in the uninduced cell cultures. For a given batch of cells with sufficient 
nutrient these observed quantities are approximately constant. For modeling purposes, we define two additional constants, the average number of copies of lytic viral DNA per cell $R_{A}$ and the average number of intracellular viral DNA copies $V_{I A}$ per lytic host cell. In terms of the uninduced model, these quantities can be written as

$$
\begin{gathered}
a_{S}=\left(\frac{H_{R}}{H_{L}+H_{R}+N}\right)_{t \rightarrow \infty} N_{r}=\left(\frac{N}{H_{L}+H_{R}+N}\right)_{t \rightarrow \infty} \\
2=\frac{\left(H_{L}+H_{R}+N\right)_{t=D}}{\left(H_{L}+H_{R}+N\right)_{t=0}} n_{T}=\left(\frac{L+R+V_{I}}{H_{L}+H_{R}+N}\right)_{t \rightarrow \infty} . \\
V_{I A}=\left(\frac{V_{I}}{H_{R}}\right)_{t \rightarrow \infty} \quad R_{A}=\left(\frac{R}{H_{R}}\right)_{t \rightarrow \infty}
\end{gathered}
$$

Using the above definitions and the uninduced equations (3), we obtain expressions for the remaining unknown model parameters in terms of the quantities $a_{s}, N_{r}, D_{p}, n_{T}, V_{I A}$, and $R_{A}$ and the model parameters $\gamma_{L}$ and $\mu$. In particular, we find that

$$
\begin{aligned}
& d_{L}=\frac{\ln (2) / D_{p}+\mu N_{r}}{1-a_{S}-N_{r}}-\gamma_{L} \\
& c=\frac{\ln (2)}{a_{S} V_{I A} D_{p}}\left(N_{r}-1\right)+\frac{\gamma_{L}}{a_{S} V_{I A}}\left(1-a_{S}-N_{r}\right) \\
& a_{0}=\gamma_{L}-\frac{\ln (2)}{D_{p}} \\
& p=\frac{R_{A}}{T V_{I A}}-\frac{\left(1-a_{S}-N_{r}\right)}{a_{S}}\left(\gamma_{L}-\frac{\ln (2)}{D_{p}}\right) \\
& \kappa=\left(\frac{\gamma_{L}-\ln (2) / D_{p}}{a_{S} R_{A}}\right)\left(R_{A}-N_{r} R_{A}-n_{T}+a_{S} V_{I A}\right)+\frac{1}{T} \\
& n=\frac{n_{T}-a_{S}\left(R_{A}+V_{I A}\right)}{1-a_{S}-N_{r}} .
\end{aligned}
$$

Values for the quantities $\gamma_{L}, \mu, a_{s}, N_{r}, D_{p}$, and $n_{T}$ can be obtained from the experimental literature [10,20,22,23,24,26,32,37], leaving $R_{A}$ and $V_{I A}$ as the only unknown parameters from the uninduced model. However, given the relations (8), $R_{A}$ and $V_{I A}$ are constrained by the other parameter values if we require positive parameter values. Values for $R_{A}$ and $V_{I A}$ are chosen to lie within the constrained range of values.

\section{Numerical Results}

In this section, we compare our reactivation model to two sets of experimental data from the literature that describe reactivation of latent virus in BCBL-1 cells. In particular, we compare our model to longitudinal cell viability data following chemical induction.

\subsection{Uninduced Case}

We first present results of a simulation for the uninduced case modeled by system (3). The observed fraction of nonviable cells $N_{r}$ (in the uninduced case) is $N_{r}=0.077$ and 0.16 for the data of Zoeteweij, et al., and Yu, et al., respectively. For the other constants we choose values from within the ranges reported in the literature: $a_{s}=0.02, D_{p}=24 \mathrm{hr}$. In addition, we choose $n_{T}=69$ and 68 for modeling the data of Zoeteweij, et al., and Yu, et al., respectively. In this 
way we have $n=10$ for both sets of data. In Table 1 we tabulate the parameter values used in simulations.

The initial condition for all compartments is zero, except for compartments $H_{L}$ and $L$, which have initial conditions of $1 \times 10^{6}$ and $1 \times 10^{7}$. Figure 3 depicts the percentage of nonviable cells $N /\left(H_{L}+H_{R}+N\right)$ and spontaneously reactivated cells $H_{R} /\left(H_{L}+H_{R}+N\right)$ from simulations using equations (3) and the parameter values in Table 1. In Fig. 3 we can see that, by 1000 hours, the percentage of nonviable cells and spontaneously reactivated cells asymptotically reach the specified equilibrium values of $a_{s}=2 \%$ and $N_{r}=7.7 \%$ for the data of Zoeteweij, et al., and $a_{s}=2 \%$ and $N_{r}=16 \%$ for the data of $\mathrm{Yu}$, et al. By equilibrium we mean that, although the cell culture is growing exponentially, certain characteristic properties related to the ratios of model compartments eventually become the constants in (7).

In Fig. 4 we can see a similar equilibration of the average number of lytic and intracellular DNA copies per lytically infected host cell, $R / H_{R}$ and $V_{I} / H_{R}$, respectively. In Fig. 4 we can see that, by 1000 hours, the quantities $R / H_{R}$ and $V_{I} / H_{R}$ have reached the specified equilibrium values of $R_{A}=2500$ and $V_{I A}=500$, respectively. The equilibrated simulations for the uninduced model approximate the properties of the uninduced cell cultures that are subsequently used in induction experiments.

\subsection{Induced Case}

Next we report on simulations for the induced equations (5). In the case of those parameters that are common to both the uninduced and induced models we use the same values as in the previous simulation (Table 1). The initial conditions for the induced model are $H_{L}(0)=(1-$ $\left.N_{r}-a_{s}\right) C_{0}, H_{R}(0)=a_{s} C_{0}, N(0)=N_{r} C_{0}, L(0)=n H_{L}, R(0)=R_{A} H_{R}, V_{I}(0)=V_{I A} H_{R}$, and $V_{F}(0)$ $=0$, where $C_{0}$ is the initial number of cells. The exact functional forms of the rates for induced lytic cell death $\delta_{R}(s)$ and induced reactivation of latent cells $\alpha(s)$ are not known. We first choose simple affine functions $\alpha(s)=\alpha_{0}+\alpha_{c} s$ and $\delta_{R}(s)=\delta_{c} s$ and find values for the function parameters by fitting longitudinal experimental data on BCBL-1 cell viability from Zoeteweij, et al., [37] and $\mathrm{Yu}$, et al., [36], separately. The parameter fitting is accomplished by forming an ordinary least squares inverse problem as described in the Appendix and then estimating the parameters using a Nelder-Mead algorithm. Standard errors are calculated, the details of which are also given in the Appendix. Estimated values for the parameters are insensitive to the initial values that seed the optimization algorithm.

Figure 5 compares cell viability predicted from simulations with data from both experimental groups, using the estimated parameters for $\delta_{c}$ and $\alpha_{c}$ obtained by the ordinary least squares estimation techniques. Cell viability is defined as $\left(H_{L}+H_{R}\right) /\left(H_{L}+H_{R}+N\right)$. In the uninduced case, $\left(H_{L}+H_{R}\right) /\left(H_{L}+H_{R}+N\right)=1-N_{r}$. Estimated parameter values are reported in Table 1 and 2. Some of the model parameters differ between the two groups because of differences in uninduced cell viability for the two groups (92\% versus 84\%, approximately). From Fig. 5 it can be seen that the simulations for the induced model qualitatively match the behavior of the experimental data.

In Fig. 6 we plot the normalized number of virions $\left(V_{F} / C_{0}\right)$ produced as a function of time for different butyrate concentrations. Yu, et al., [36] observe in their experiments that high concentrations of butyrate $(1.5$ and $3 \mathrm{mM}$ ) greatly increase lytic activity, but also significantly increase cell death. The end result is that, even after 5 days, very few virions are produced because of massive amounts of cell death before the end of the lytic program. This is contrasted by observations at smaller concentrations of butyrate $(\leq 0.3 \mathrm{mM})$, where much less cell death is seen and there is significant secretion of virions. In Fig. 6 it can be seen that there is approximately a three to four-fold increase in free virion produced at $0.3 \mathrm{mM}$ concentration of butyrate as compared to the $3 \mathrm{mM}$ concentration. 
The results of Fig. 6 illustrate that there is an optimal dosage threshold that maximizes viral production. Below the optimal dose there is not enough reactivation of latent virus and above the optimal dose the inducer kills the lytically replicating cells before virus is produced. In Fig. 7 we plot the optimal butyrate dose that maximizes virion production as a function of the induction time. These results are obtained numerically from simulations of the induced equations (5) with the two sets of parameters for the data of Zoetewij et al. (dashed line) and Yu et al. (solid line).

Table 2 summarizes the estimated parameters, standard errors, and confidence intervals obtained from fitting the induced equations (5) with the parameter functions $\alpha(s)=\alpha_{0}+\alpha_{c} s$ and $\delta_{R}(s)=\delta_{c} s$ to both sets of data. Table 2 shows that the estimated parameter values for both groups are within an order of magnitude of each other. Differences between the parameter values may reflect differences in the cell growth and maintenance conditions or differences in experimental measurement techniques. Even in the uninduced case, there is a difference in the cell viability for both groups, with $N_{r}=0.077$ for the data of Zoeteweij, et al., and $N_{r}=0.16$ for the data of Yu, et al. In addition, Zoeteweij, et al., measure cell viability using Dead Red staining and flow cytometry, while $\mathrm{Yu}$, et al., measure cell viability with trypan blue staining and counting on a haemocytometer. In Table 2, it can also be seen that the standard errors for the reactivation rate constants $\alpha_{c}$ are at least an order of magnitude less than the parameter values. However, the standard errors for the induced death rate constants $\delta_{c}$ are the same order of magnitude as the parameter values, providing us with less confidence in values obtained for $\delta_{c}$.

\section{Discussion}

In other simulations, we used different functional forms for $\alpha(s)$ and $\delta_{R}(s)$, including MichaelisMenton and sigmoid functions, but we found that the fits of the induced equations to cell viability data were relatively insensitive to more complicated functional forms (data not shown) and that reasonable fits to cell viability data were obtained by assuming simple linear functions. However, with more data, especially with data for viral DNA compartments, we expect to be able to determine optimal functional forms for $\alpha(s)$ and $\delta_{R}(s)$, for example, combinations of linear, Michaelis-Menton, and sigmoid functions. Alternatively, instead of fixing the functional form of $\alpha(s)$ and $\delta_{R}(s)$ a priori in parametric form, we could estimate the shape of the functional form itself using approximation by piece-wise linear splines or other approximations as has been successfully done in other problems in, for example, [1,2].

Even though this preliminary model yields good qualitative agreement with cell viability data, additional experimental data is needed to adequately evaluate model predictions for the viral compartments of the model. Development and evaluation of this model has highlighted the need for different types of data than is often gathered in typical experiments. For example, many experiments measure only at a single time point after induction, but evaluation of a dynamic model such as the one presented here requires data at multiple time points. In addition, many experiments produce only qualitative data, for example Western blot data, or relative data, such as the fold-increase of RNA production often measured in real-time PCR, while evaluation of the model presented here requires quantitative data. Finally, establishing a workable mathematical model may require additional experiments to determine parameter values (e.g., $R_{A}$ and $V_{I A}$ ) and validate the model that are not necessarily of biological interest to experimentalists. Quantitative experimental measurements of cell-associated DNA ( $L+R$ $\left.+V_{I}\right)$ and virion production $\left(V_{F}\right)$ using real-time PCR (with a standard curve calibration) at a series of time points after induction (or without induction) would provide the type of quantitative data that is needed to test the viral dynamics of the model. 
Presently, experimental measurements of the viral load $n_{T}=\left(L+R+V_{I}\right) /\left(H_{L}+H_{R}+N\right)$ are reported in the literature to be in the range of 50-70. Our model predicts that the number of latent copies of virus per uninduced host cell $\left(n=L / H_{L}\right)$ is much less than this (we estimate $n=10$ ). The experimental measurements proposed above combined with quantitative measurements of $n$ could be used to determine the parameters $R_{A}$ and $V_{I A}$ in uninduced BCBL-1 cells.

Once established, it may be possible to extend the model to predict gene expression data, which is of more interest to experimentalists. Instead of a single viral compartment $R$ to quantify copies of viral DNA in the lytic program, we could modify the model to describe Immediate Early, Early, and Late gene expression (RNA) by dividing the $R$ compartment into three compartments $R_{1}, R_{2}$, and $R_{3}$. By having model compartments that quantify RNA production or promoter activity from genes representative of each stage of the lytic cycle, we might predict viral reactivation in more detail and compare to gene expression data from experiments. A model that subdivides the $R$ compartment might also work better if there are underlying biological delays, due to the ordered cascade of gene expression that makes up the lytic program, that are not captured with the present model.

\section{Conclusion}

We have developed a preliminary deterministic mathematical model to describe reactivation of latent virus by chemical inducers. In particular, we apply this model to the reactivation of latent KSHV in BCBL-1 cell cultures with butyrate as the inducing agent. We first estimate parameters for our uninduced model from physiological considerations and known properties of these exponentially growing, uninduced cell cultures. We then extend the model to describe chemically induced KSHV reactivation in latently infected BCBL-1 cells. Additional parameters that describe induction are determined from fits to experimental data available in the literature. Our model provides good agreement with two independent sets of experimental data. While this preliminary model yields good qualitative agreement with cell viability data for KSHV induced by butyrate, it also strongly suggests the need for further experiments designed explicitly to support model development and validation in providing not only more but also additional types of longitudinal data.

This model could be applied to other inducers, particularly other HDAC inhibitors (e.g., SFA's). Application of the model to other SFA's would be the next step towards developing a polymicrobial model for viral reactivation by the metabolic end products of gram negative bacteria in an in vivo environment. The model could also be applied to inducers such as TPA, which is commonly used in KSHV induction experiments but does not induce the same level of cell death as butyrate. For this reason and because TPA causes viral reactivation through a cell-signalling mechanism rather than HDAC inhibition, the form of the induction parameters $\alpha(s)$ and $\delta_{R}(s)$ might differ from the simple affine functions used for butyrate.

Many other latent viruses are induced to replication via HDAC inhibition and are responsive to agents like sodium butyrate (e.g., EBV, HCMV, HSV, HIV, Adenovirus, HPV, and HTLV1). Application of this model with butyrate as an inducer to other cell lines with latent virus (e.g., EBV in the B95-8 cell line) could provide information about the similarities and differences among these latent virus systems.

\section{Acknowledgements}

This research was supported in part by the US Air Force Office of Scientific Research under grant AFOSR FA9550-04-1-0220, in part by the Joint DMS/NIGMS Initiative to Support Research in the Area of Mathematical Biology under grant 1R01GM67299-01, in part by the National Science Foundation under grant DMS-0112069 to the Statistical and Applied Mathematical Sciences Institute (SAMSI), and in part by the UNC Center for AIDS Research under grant K23 DE 00460-01. 


\section{References}

1. Adams, BM. PhD Thesis. Center for Research in Scientific Computation: Mathematics Department, North Carolina State University; 2005. Non-parametric Parameter Estimation and Clinical Data Fitting With a Model of HIV Infection.

2. Banks, HT.; Kunisch, K. Estimation Techniques for Distributed Parameter Systems. Birkhaüser, Boston: 1989.

3. Banks HT, Bortz DM, Holte SE. Incorporation of variability into the mathematical modeling of viral delays in HIV infection dynamics. Mathematical Biosciences 2003;183:63-91. [PubMed: 12604136]

4. Banks HT, Nguyen HK. Sensitivity of dynamical system to Banach space parameters. CRSC Tech Rep., CRSC-TR05-13, N.C. State University. J Math Anal Appl. 2005 Feb;in press

5. Bechtel JT, Liang YY, Hvidding J, Ganem D. Host range of Kaposi's sarcoma-associated herpesvirus in cultured cells. Journal of Virology 2003;77(11):6474-6481. [PubMed: 12743304]

6. Bernhard D, Ausserlechner MJ, Tonko M, Lo²er M, Hartmann BL, Csordas A, Kofler R. Apoptosis induced by the histone deacetylase inhibitor sodium butyrate in human leukemic lymphoblasts. FASEB Journal 1999;13(14):1991-2001. [PubMed: 10544182]

7. Blower SM, Porco TC, Darby G. Predicting and preventing the emergence of antiviral drug resistance in HSV-2. Nature Medicine 1998;4(6):673-678.

8. Cannon JS, Ciufo D, Hawkins AL, Griffin CA, Borowitz MJ, Hayward GS, Ambinder RF. A new primary effusion lymphoma-derived cell line yields a highly infectious Kaposi's sarcoma herpesviruscontaining supernatant. Journal of Virology 2000;74(21):10187-10193. [PubMed: 11024147]

9. Casella, G.; Berger, RL. Statistical Inference. Duxbury; California: 2002.

10. Chan SR, Bloomer C, Chandran B. Identification and characterization of human herpesvirus-8 lytic cycle-associated ORF59 protein and the encoding cDNA by monoclonal antibody. Virology 1998;240(1):118-126. [PubMed: 9448696]

11. Csordas, A. Toxicology of butyrate and short-chain fatty acids. In: Hill, MJ., editor. Role of Gut Bacteria in Human Toxicology and Pharmacology. Taylor \& Francis; London: 1995.

12. Davidian, M.; Giltinan, DM. Nonlinear Models for Repeated Measurement Data. Chapman and Hall/ CRC; Boca Raton: 1995.

13. Deutsch E, Cohen A, Kazimirsky G, Dovrat S, Rubinfeld H, Brodie C, Sarid R. Role of protein kinase C $\delta$ in reactivation of Kaposi's sarcoma-associated herpesvirus. Journal of Virology 2004;78(18): 10187-10192. [PubMed: 15331751]

14. Flint, SJ.; Enquist, LW.; Racaniello, VR.; Skalka, AM. Principles of Virology. ASM Press; Washington DC: 2004.

15. Friborg J, Kong WP, Hottiger MO, Nabel GJ. p53 inhibition by the LANA protein of KSHV protects against cell death. Nature 1999;402(6764):889-894. [PubMed: 10622254]

16. Garnett GP, Grenfell BT. The epidemiology of varicella zoster virus-infections - a mathematicalmodel. Epidemiology and Infection 1992;108(3):495-511. [PubMed: 1318218]

17. Gallant, AR. Nonlinear Statistical Models. John Wiley \& Sons, Inc; New York: 1987.

18. Izumiya Y, Lin SF, Ellison TJ, Levy AM, Mayeur GL, Izumiya C, Kung HJ. Cell cycle regulation by Kaposi's sarcoma-associated herpesvirus K-bZIP: Direct interaction with cyclin-CDK2 and induction of G(1) growth arrest. Journal of Virology 2003;77(17):9652-9661. [PubMed: 12915577]

19. Jennrich RI. Asymptotic properties of non-linear least squares estimators. Ann Math Statist 1969;40:633-643.

20. Klass CM, Krug LT, Pozharskaya VP, Offermann MK. The targeting of primary effusion lymphoma cells for apoptosi by inducing lytic replication of human herpesvirus 8 while blocking virus production. Blood 2005;105(10):4028-4034. [PubMed: 15687238]

21. Kepler, GM.; Nguyen, HK.; Webster-Cyriaque, J.; Banks, HT. A dynamic model for induced reactivation of latent virus. CRSC Technical Reports, CRSC-TR05-44; 2005.

22. Lallemand F, Desire N, Rozenbaum W, Nicolas JC, Marechal V. Quantitative analysis of human herpesvirus 8 viral load using real-time PCR assay. Journal of Clinical Microbiology 2000;38(4): 1404-1408. [PubMed: 10747115] 
23. Lu M, Suen J, Frias C, Pfeiffer R, Tsai MH, Chuang E, Zeichner SL. Dissection of the Kaposi's sarcoma-associated herpesvirus gene expression program by using the viral DNA replication inhibitor cidofovir. Journal of Virology 2004;78(24):13637-13652. [PubMed: 15564474]

24. Lukac DM, Renne R, Kirshner JR, Ganem D. Reactivation of Kaposi's sarcoma-associated herpesvirus infection from latency by expression of the ORF50 transactivator, a homolog of the EBV R protein. Virology 1998;252(2):304-312. [PubMed: 9878608]

25. Lukac DM, Kirshner JR, Ganem D. Transcriptional activation by the product of open reading frame 50 of Kaposi's sarcoma-associated herpesvirus is required for lytic viral reactivation in B cells. Journal of Virology 1999;73(11):9348-9361. [PubMed: 10516043]

26. Renne R, Lagunoff M, Zhong WD, Ganem D. The size and conformation of Kaposi's sarcomaassociated herpesvirus (human herpesvirus 8) DNA in infected cells and virions. Journal of Virology 1996;70(11):8151-8154. [PubMed: 8892944]

27. Renne R, Zhong WD, Herndier B, McGrath M, Abbey N, Kedes D, Ganem D. Lytic growth of Kaposi's sarcoma-associated herpesvirus (human herpesvirus 8) in culture. Nature Medicine 1996;2 (3):342-346.

28. Rothwell R, Morris T, Granger D, Dominick L, Picot B, Duus K, Webster-Cyriaque J. Pathogenic aneaorobic bacteria cause epigenetic changes that result in viral reactivation. submitted

29. Said JW, Chien K, Tasaka T, Koeffler HP. Ultrastructural characterization of human herpesvirus 8 (Kaposi's sarcoma-associated herpesvirus) in Kaposi's sarcoma lesions: Electron microscopy permits distinction from cytomegalovirus (CMV). Journal of Pathology 1997;182(3):273-281. [PubMed: 9349229]

30. Sawtell NM. Comprehensive quantification of herpes simplex virus latency at the single-cell level. Journal of Virology 1997;71(7):5423-5431. [PubMed: 9188614]

31. Seber, GAF.; Wild, CJ. Nonlinear Regression. John Wiley \& Sons, Inc; New York: 1989.

32. Tinari A, Monini P, Marchetti M, Ammendolia MG, Leone P, Ensoli B, Superti F. Lytic growth of human herpesvirus 8: Morphological aspects. Ultrastructural Pathology 2000;24:301-310. [PubMed: 11071568]

33. Wang GY, Krueger GRF, Buja LM. Mathematical model to simulate the cellular dynamics of infection with human herpesvirus-6 in EBV-negative infectious mononucleosis. Journal of Medical Virology 2003;71(4):569-577. [PubMed: 14556271]

34. Wu FY, Ahn JH, Alcendor DJ, Jang WJ, Xiao JS, Hayward SD, Hayward GS. Origin-independent assembly of Kaposi's sarcoma-associated herpesvirus DNA replication compartments in transient cotransfection assays and association with the ORF-K8 protein and cellular PML. Journal of Virology 2001;75(3):1487-1506. [PubMed: 11152521]

35. Wu FY, Tang QQ, Chen HL, ApRhys C, Farrell C, Chen JM, Fujimuro M, Lane MD, Hayward GS. Lytic replication-associated protein (RAP) encoded by Kaposi sarcoma-associated herpesvirus causes p21(CIP-1)-mediated G(1) cell cycle arrest through CCAAT/enhancer-binding protein-alpha. Proceedings of the National Academy of Sciences USA 2002;99(16):10683-10688.

36. Yu Y, Black JB, Goldsmith CS, Browning PJ, Bhalla K, Offermann MK. Induction of human herpesvirus-8 DNA replication and transcription by butyrate and TPA in BCBL-1 cells. Journal of General Virology 1999;80:83-90. [PubMed: 9934688]

37. Zoeteweij JP, Eyes ST, Orenstein JM, Kawamura T, Wu LJ, Chandran B, Forghani B, Blauvelt A. Identification and rapid quantification of early- and late-lytic human herpesvirus 8 infection in single cells by flow cytometric analysis: Characterization of antiherpesvirus agents. Journal of Virology 1999;73(7):5894-5902. [PubMed: 10364341]

\section{Appendix}

In this appendix we discuss the asymptotic theory used to compute the standard errors and confidence intervals in Table 2 of Section 6 . We first give a general summary of the theory.

We assume $N^{*}$ scalar longitudinal/inducer level observations (time/inducer series of numbers or ratios of numbers of cells as described below) are represented by the statistical model 


$$
Y_{j} \equiv f_{j}\left(\theta_{0}\right)+\varepsilon_{j}, j=1,2, \ldots N^{*},
$$

where $f_{j}\left(\theta_{0}\right)$ is the model for the observations in terms of the state variables and $\theta_{0} \in \mathbb{R}^{m}$ is a "set" of theoretical "true" parameter values (assumed to exist in a standard statistical approach). We assume for our statistical model of the observation or measurement process (9) that the errors $\varepsilon_{j}, j=1,2, \ldots, N^{*}$, are independent identically distributed (i.i.d.) random variables with mean $E\left[\varepsilon_{j}\right]=0$ and constant variance $\operatorname{var}\left[\varepsilon_{j}\right]=\sigma_{0}^{2}$, where of course $\sigma_{0}^{2}$ is unknown (standard residual plots with the data used in our simulation suggested this assumption of constant variance). We then have that the observations $Y_{j}$ are i.i.d. with mean $E\left[Y_{j}\right]=f_{j}\left(\theta_{0}\right)$ and variance $\operatorname{var}\left[Y_{j}\right]=\sigma_{0}^{2}$.

We consider estimation of parameters using an ordinary least squares (OLS) approach. Thus we seek to use data $\left\{y_{j}\right\}$ for the observation process $\left\{Y_{j}\right\}$ with the model to seek a value $\theta$ that minimizes

$$
J(\theta)=\sum_{j=1}^{N^{*}}\left|y_{j}-f_{j}(\theta)\right|^{2}
$$

Since $Y_{j}$ is a random variable, we have that the estimator $\theta_{O L S}$ is also a random variable with a distribution called the sampling distribution. Knowledge of this sampling distribution provides uncertainty information (e.g., standard errors) for the numerical values of $\theta$ obtained using a specific data set $\left\{y_{j}\right\}$ (i.e., a realization of $\left\{Y_{j}\right\}$ ) when minimizing $J(\theta)$.

Under reasonable assumptions on smoothness and regularity (the smoothness requirements for model solutions are readily verified using continuous dependence results for ordinary differential equations in our example; the regularity requirements involve, among others, conditions on how the observations are taken as sample size increases, i.e., $N^{*} \rightarrow \infty$ ), the standard nonlinear regression approximation theory ([12], [17], [19], and Chapter 12 of [31]) for asymptotic (as $N^{*} \rightarrow \infty$ ) distributions can be invoked. This theory yields that the sampling distribution $\theta(Y)$ for the estimate $\theta$, where $Y=\{Y\}_{j=1} N_{j=1}^{*}$, is approximately a $m$-multivariate Gaussian with mean $E[\theta(Y)] \approx \theta_{0}$ and covariance matrix $\operatorname{cov}[\hat{\theta}(Y)] \approx \Sigma_{0}=\sigma_{0}^{2}\left[\chi^{T}\left(\theta_{0}\right) \chi\left(\theta_{0}\right)\right]^{-1}$. Here $\chi(\theta)=F_{\theta}(\theta)$ is the $N^{*} \times m$ sensitivity matrix with elements

$$
\chi_{j k}(\theta)=\frac{\partial f_{j}^{(\theta)}}{\partial \theta_{k}} \text { and } F_{\theta}(\theta) \equiv\left(f_{1 \theta^{(\theta)}, \ldots, f_{N * \theta^{(\theta))}} T}\right.
$$

That is, for $N^{*}$ large, the sampling distribution approximately satisfies

$$
\hat{\theta}_{O L S}(Y) \sim \mathrm{N}_{m}\left(\theta_{0}, \sigma_{0}^{2}\left[\chi^{T}\left(\theta_{0}\right) \chi\left(\theta_{0}\right)\right]^{-1}\right):=\mathrm{N}_{m}\left(\theta_{0}, \Sigma_{0}\right)
$$

The elements of the matrix $=\left(\chi_{j k}\right)$ can be estimated using the forward difference

$$
\chi_{j k}(\theta)=\frac{\partial f_{j}(\theta)}{\partial \theta_{k}} \approx \frac{f_{j}\left(\theta+h_{k}\right)-f_{j}(\theta)}{\left|h_{k}\right|},
$$

where $h_{k}$ is an $m$-vector with nonzero entry in only the $k^{\text {th }}$ component, or using sensitivity equations (see [4] and the references therein). For our efforts here we chose the sensitivity 
equation approach as explained below. Since $\theta_{0}, \sigma_{0}$ are not known, we must approximate them in $\Sigma_{0}=\sigma_{0}^{2}\left[\chi^{T}\left(\theta_{0}\right) \chi\left(\theta_{0}\right)\right]^{-1}$. For this we follow standard practice and use the approximation

$$
\Sigma_{0} \approx \Sigma(\hat{\theta})=\hat{\sigma}^{2}\left[\chi^{T}(\hat{\theta}) \chi(\hat{\theta})\right]^{-1}
$$

where $\theta$ is the parameter estimate obtained, and the approximation $\hat{\sigma}^{2}$ to $\sigma_{0}^{2}$ is given by

$$
\sigma_{0}^{2} \approx \hat{\sigma}^{2}=\frac{1}{N^{*}-m} \sum_{j=1}^{N^{*}}\left|y_{j}-f_{j}(\hat{\theta})\right|^{2}
$$

Standard errors to be used in confidence interval calculations are thus given by $S E_{k}(\hat{\theta})=\sqrt{\Sigma_{k k}(\hat{\theta})}, k=1,2, \ldots, m($ see $[9])$.

In the induced case example of Section 4, we consider the parametric functional forms $\delta_{R}(s)$ $=d_{C} s$ and $\alpha(s)=\alpha_{c} s+\alpha_{0}$. If we let $x=\left(H_{L}, H_{R}, N, L, R, V_{I}, V_{F}\right)^{T}$ and denote $\theta=\left(\delta_{c}, \alpha_{c}\right)$, then the differential equations in the induced case can be written in a general form

$$
\begin{aligned}
x & =g(t, x, s, \theta) \\
x(0) & =x_{0},
\end{aligned}
$$

where $g: \mathbb{R}_{+} \times \mathbb{R}^{n^{*}} \times \mathbb{R}_{+} \times \mathbb{R}^{m} \rightarrow \mathbb{R}^{n^{*}}$ for $n^{*}=7, m=2$, and $x_{0}=\left(H_{L 0}, H_{R 0}, N_{0}, L_{0}, R_{0}, V_{I 0}\right.$, $\left.V_{F 0}\right)^{T}$. Since the experimental data are given in percentage of viable cells, we define the outputs of the model

$$
f(t, s, \theta)=\left[\frac{V_{\text {total }}(t, s, \theta)-N(t, s, \theta)}{V_{\text {total }}(t, s, \theta)}\right], \quad t, s \geq 0
$$

where $V_{\text {total }}=H_{L}+H_{R}+N$. In each parameter fit, we use data that is longitudinal (taken at $\left.t_{k}\right)$ and across several levels $s_{i}$ of inducer. This is indexed by $\tau_{j}=\left(t_{k}, s_{i}\right)$ for $k=1, \ldots, K, i=1$, $\ldots, I$, and observations $y_{j}$ for the model values $f_{j}(\theta)=f\left(t_{k}, s_{i}, \theta\right), j=1, \ldots, N^{*}=K I$. Then, we construct the OLS estimator by minimizing the cost criterion (10) where $\left\{y_{j}\right\}$ denotes the experimental data (in the data of Section 4 we had $N^{*}=15$ or 16 resulting from $K=4$ and $I=$ 4 - see Fig. 5). For the optimization in $\theta$ we used the Nelder-Mead algorithm.

To compute the covariance matrix $\Sigma$ we need the sensitivity matrix $F_{\theta}$. That is, $\chi(\hat{\theta})=\frac{\partial F}{\partial \theta}(\hat{\theta})$. From the outputs defined in (12), it suffices to have the sensitivities $\frac{\partial x}{\partial \theta}$. To compute these we used the sensitivity equation method which involves solving the $n^{*} \times m$ matrix variational differential equation

$$
\frac{d}{d t}\left(\frac{\partial x}{\partial \theta}\right)=\frac{\partial g}{\partial x} \frac{\partial x}{\partial \theta}+\frac{\partial g}{\partial \theta}
$$

where the matrix coefficient and the forcing function in this equation are evaluated along solutions of the system equation (12). Note that this variational equation can be solved simultaneously (see [4] for details) with the system equation (12).

Finally, in order to compute the confidence intervals (at the 100(1-c)\% level) for the estimated parameters in our example, we define the confidence level parameters associated with the estimated parameters so that 


$$
P\left(\hat{\theta}_{k}-t_{c / 2} S E_{k}(\hat{\theta})<\theta_{k}<\hat{\theta}_{k}+t_{c / 2} S E_{k}(\hat{\theta})\right)=1-c
$$

where $c \in[0,1]$, and $t_{c / 2} \in \mathbb{R}_{+}$. For a given $c$ value (small, say $c=.05$ for $95 \%$ confidence intervals), the critical value $t_{c / 2}$ is computed from the Student's t distribution $t^{N^{*}-m}$ with $N^{*-}$ $m$ degrees of freedom since for each of the data sets available to us we have $N^{*}$ is less than 30 . The value of $t_{c / 2}$ is determined by $P\left(T \geq t_{c / 2}\right)=c / 2$ where $T \sim t^{N *-m}$. 


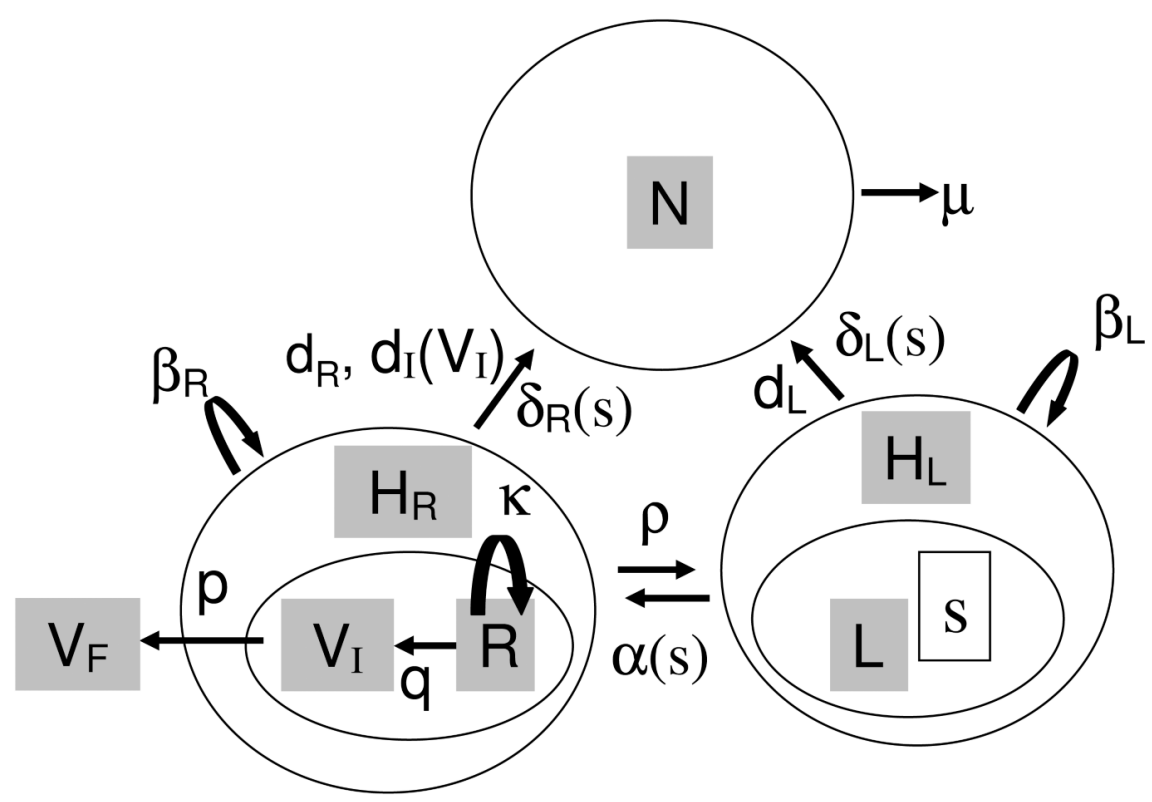

\begin{tabular}{lcl}
\hline Compartment & Symbol & Units \\
\hline Host cells (latent only) & $\mathbf{H}_{\mathrm{L}}$ & Number of cells \\
Host cells (lytic only) & $\mathbf{H}_{\mathbf{R}}$ & Number of cells \\
Nonviable cells & $\mathrm{N}$ & Number of cells \\
Latent virus & $\mathrm{L}$ & DNA copies \\
Lytic virus & $\mathrm{R}$ & DNA copies \\
Intracellular virus & $\mathrm{V}_{\mathrm{I}}$ & DNA copies \\
Free virus & $\mathrm{V}_{\mathrm{F}}$ & Number of virions \\
\hline
\end{tabular}

Fig 1.

Schematic of the modeling compartments associated with latent virus host cells $H_{L}$, lytic virus host cells $H_{R}$, and nonviable host cells $N$. Latent virus $L$ reactivates to become lytic virus $R$, either spontaneously or in response to an inducing agent $s$. Lytic virus $R$ undergoes exponential growth until it passes to the intracellular viral compartment $V_{I}$ where it is available for packaging and is released as free virions $V_{F}$. The parameters $\alpha(s)$ and $\delta_{R}(s)$ describe induced reactivation and cell death. In the absence of inducer $\delta_{R}(0)=0$ and $\alpha(0)=\alpha_{0}$, where $\alpha_{0}$ is the spontaneous reactivation rate constant. It is sometimes convenient to refer to a single parameter $\gamma_{L} \equiv \beta_{L}-d_{L}$. 


\section{The Iterative Modeling Process}

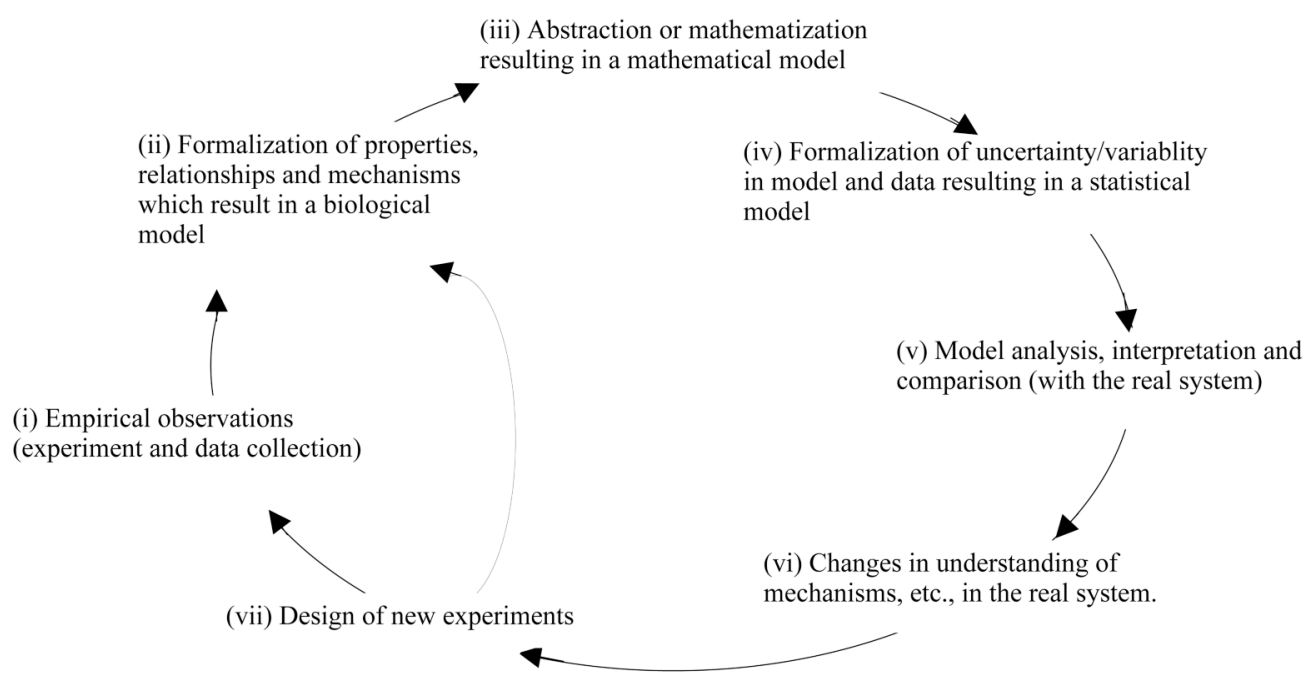

Fig 2.

Schematic of the iterative modeling process. 


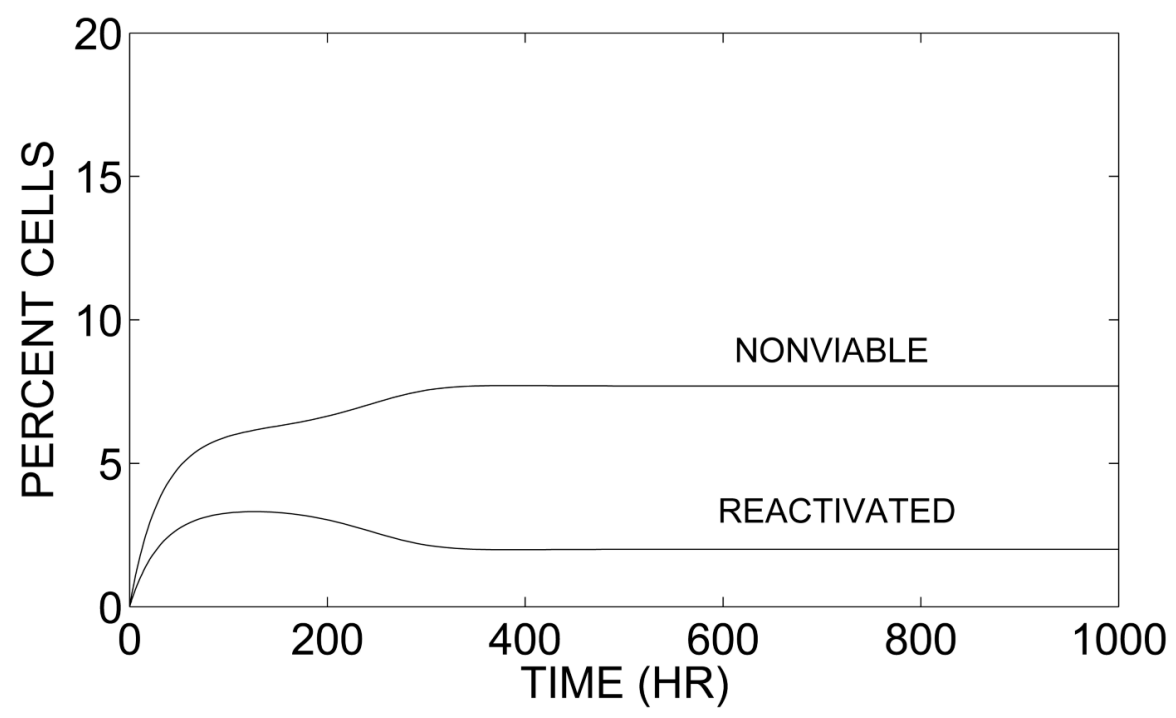

(a)

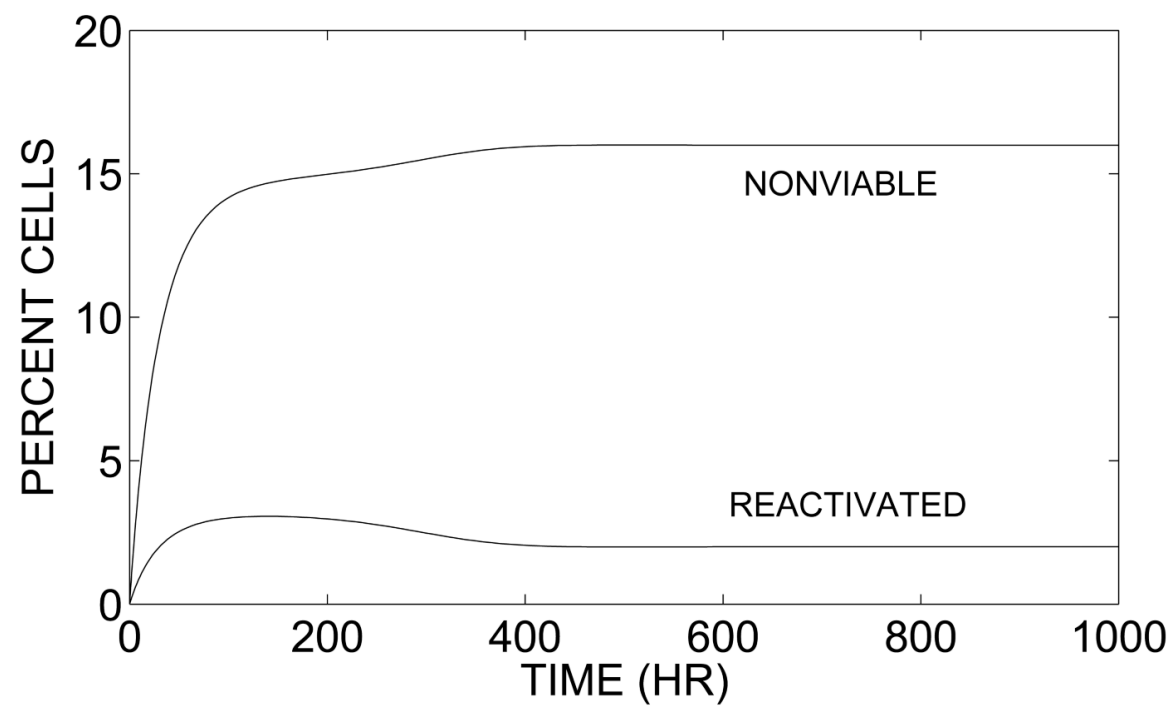

(b)

Fig 3.

The percentage of cells that are spontaneously reactivated $100 \times H_{R} /\left(H_{L}+H_{R}+N\right)$ and the percentage of nonviable cells $100 \times N /\left(H_{L}+H_{R}+N\right)$ are plotted for uninduced simulations using equations (3) and parameters from Table 1 for the data of a) Zoeteweij, et al., and b) Yu, et al. The percentage of nonviable cells and spontaneously reactivated cells asymptotically reach the specified equilibrium values of $a_{s}=2 \%$ and $N_{r}=7.7 \%$ for the data of Zoeteweij, et $a l$., and $a_{s}=2 \%$ and $N_{r}=16 \%$ for the data of Yu, et al. 


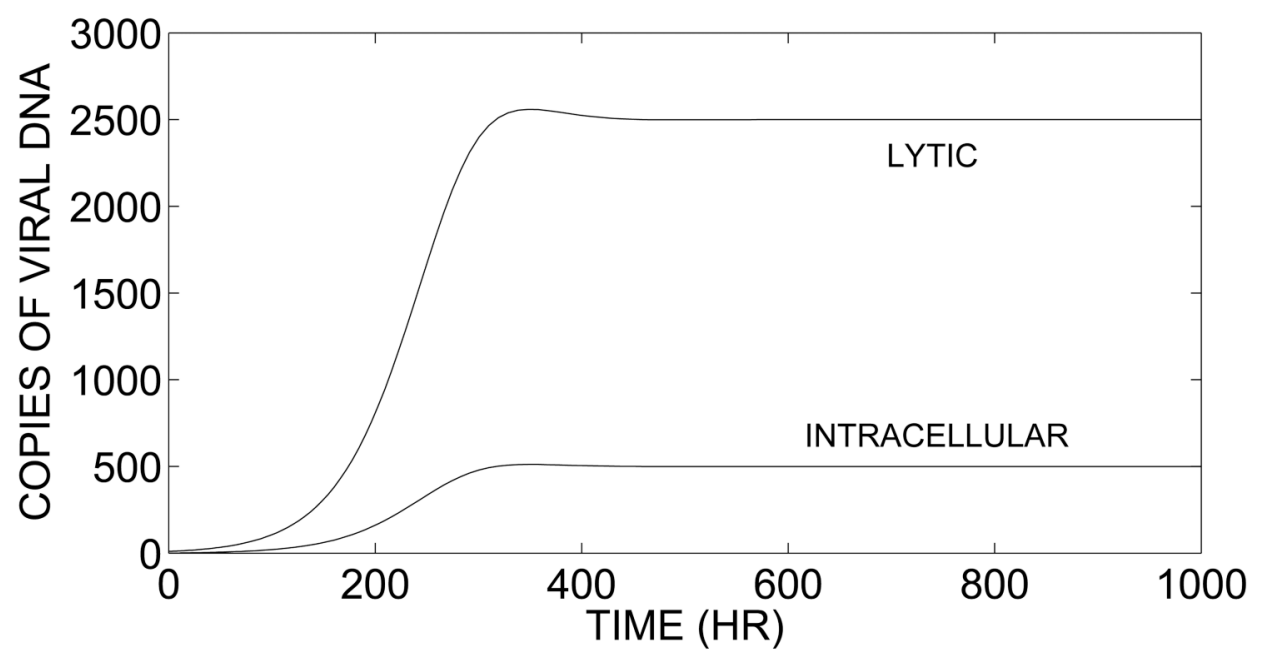

(a)

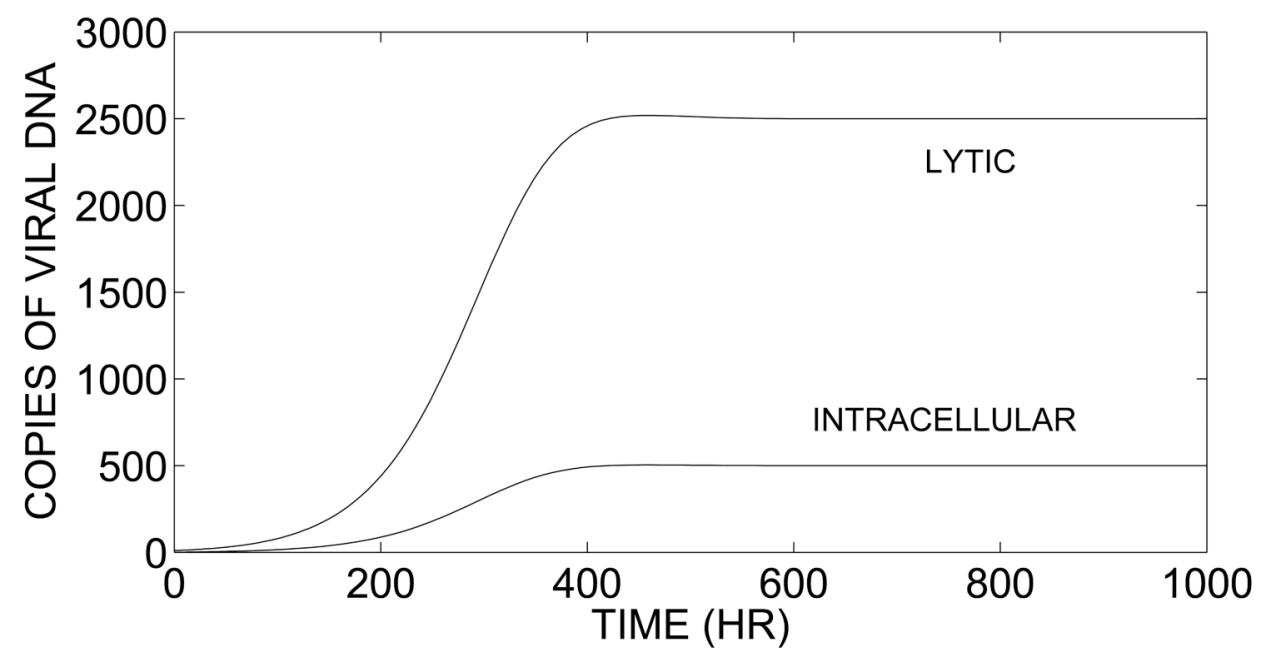

(b)

Fig 4.

Uninduced simulations using equations (3) and parameters from Table 1 for the data of a) Zoeteweij, et al., and b) Yu, et al. The plots show the average number of lytic viral DNA copies $R / H_{R}$ and the average number of intracellular viral DNA copies $V_{I} / H_{R}$ per lytically infected host cell. The quantities $R / H_{R}$ and $V_{I} / H_{R}$ asymptotically reach the specified equilibrium values of $R_{A}=2500$ and $V_{I A}=500$, respectively 


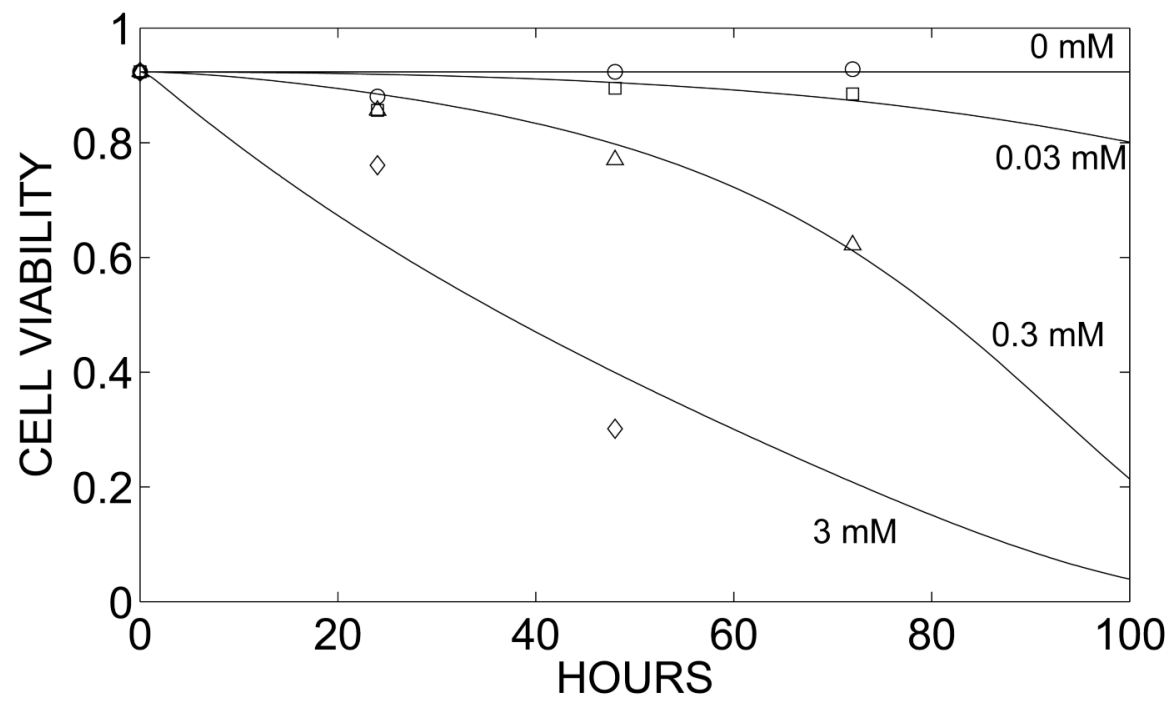

(a)

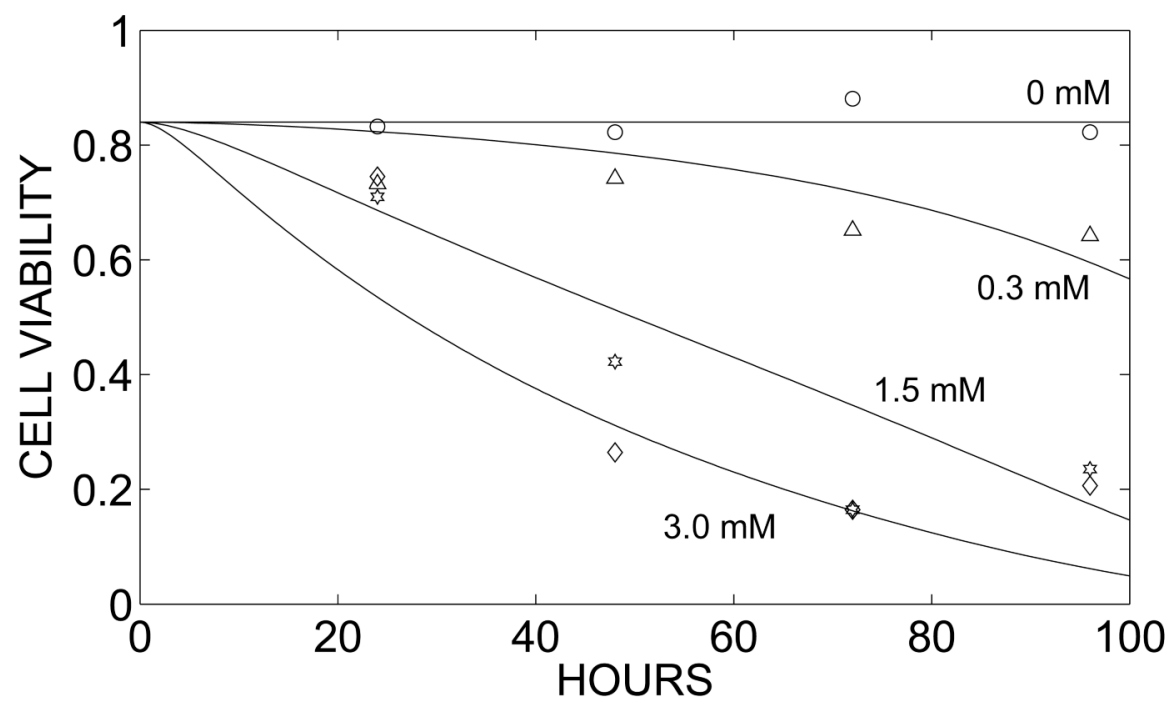

(b)

Fig 5.

Comparison of cell viability measurements (symbols) with simulations (solid lines) using induced equations (5) and fitted parameters for linear functions $\alpha(s)$ and $\delta_{R}(s)$ : a) Zoeteweij, et al., circles $0 \mathrm{mM}$, squares $0.03 \mathrm{mM}$, triangles $0.3 \mathrm{mM}$, and diamonds $3 \mathrm{mM}, \alpha_{c}=0.551$, $\delta_{c}=5.13 \times 10^{-3}$ and b) $\mathrm{Yu}$, et al., circles $0 \mathrm{mM}$, triangles $0.3 \mathrm{mM}$, stars $1.5 \mathrm{mM}$, and diamonds $3 \mathrm{mM}, \alpha_{c}=0.140, \delta_{c}=6.76 \times 10^{-3}$. Cell viability is defined as $\left(H_{L}+H_{R}\right) /\left(H_{L}+H_{R}+N\right)$. In the uninduced case, $\left(H_{L}+H_{R}\right) /\left(H_{L}+H_{R}+N\right)=1-N_{r}$. 


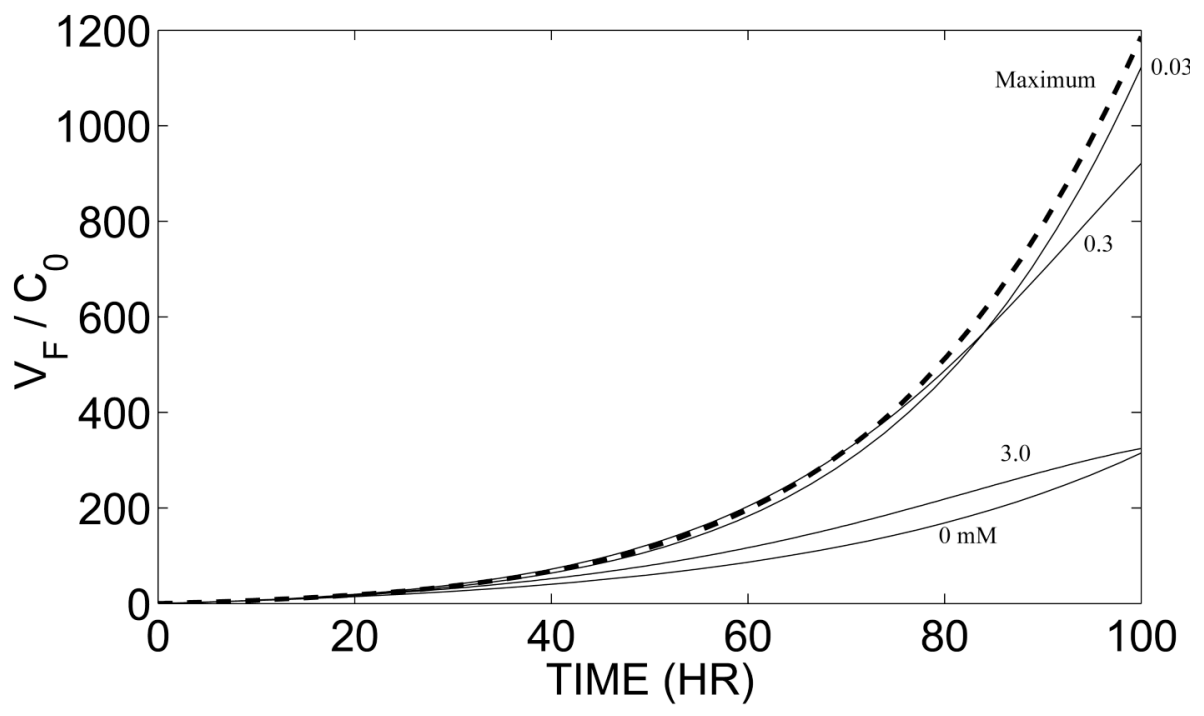

(a)

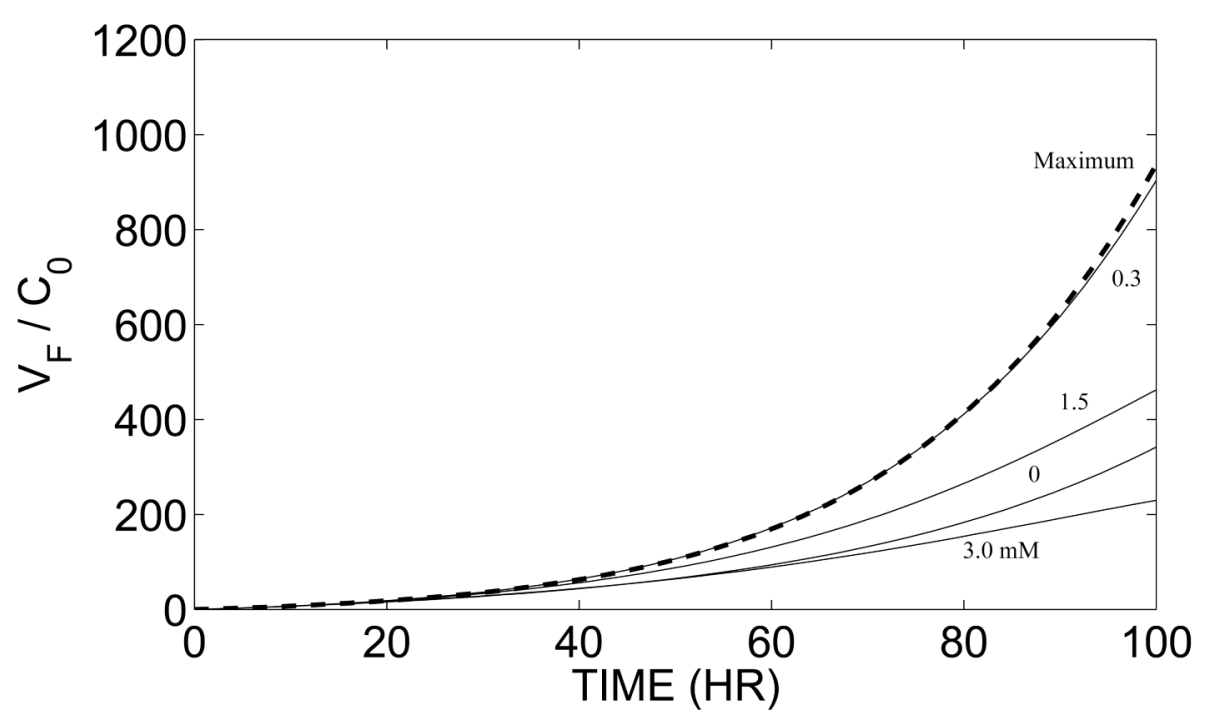

(b)

Fig 6.

The number of free virions produced per initial cell $\left(V_{F} / C_{0}\right)$ is plotted as a function of time for different butyrate doses. The data is obtained from simulations of the induced equations (5) and (6) using parameters for linear functions $\alpha(s)$ and $\delta_{R}(s)$ fitted to experimental data from a) Zoeteweij, et al., and b) Yu, et al. The dashed lines represent the maximum virion production at 100 hours using the optimal doses of a) 0.0591 and b) $0.177 \mathrm{mM}$. See Fig. 7 and the discussion below. 


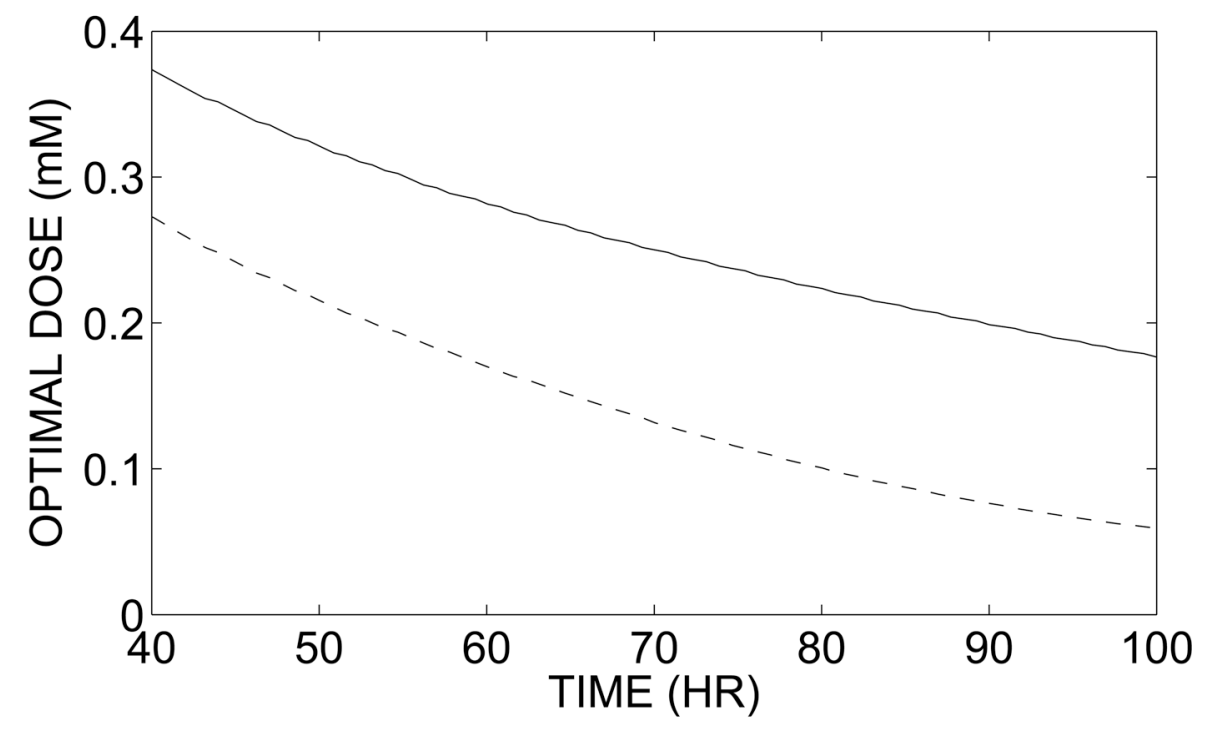

Fig 7.

The optimal butyrate concentration to maximize the quantity of virions produced is plotted as a function of the elapsed induction time. Optimal values are obtained numerically from simulations with the induced equations (5) and (6) and parameters values (Table 1) obtained from fits to data from Zoeteweij et al. (dashed line) and Yu et al. (solid line). 
Table 1

Parameters from the uninduced model (3) are calculated from (8) with constants $a_{s}=0.02, N_{r}=0.077$ or 0.16 , $D_{p}=24 \mathrm{hr}, n=10, V_{I A}=500$, and $R_{A}=2500$. Parameters from the induced model (5) are obtained from fits to experimental data.

\begin{tabular}{|c|c|c|c|c|}
\hline Parameter & Symbol & Zoeteweij, et al., data & Yu, et al., data & Units \\
\hline Net growth of latent host cells & $\gamma_{L}$ & $3.00 \times 10^{-2}$ & $3.00 \times 10^{-2}$ & $\mathrm{hr}^{-1}$ \\
\hline Nonviable cell degradation & $\mu$ & $1.00 \times 10^{-5}$ & $1.00 \times 10^{-5}$ & $\mathrm{hr}^{-1}$ \\
\hline Natural death of latent host cells & $d_{L}$ & $1.98 \times 10^{-3}$ & $5.22 \times 10^{-3}$ & $\mathrm{hr}^{-1}$ \\
\hline Spontaneous reactivation of latent host cells & $\alpha_{0}$ & $1.12 \times 10^{-3}$ & $1.12 \times 10^{-3}$ & $\mathrm{hr}^{-1}$ \\
\hline Cell death due to viral lysis & $c$ & $4.33 \times 10^{-5}$ & $3.40 \times 10^{-5}$ & $\mathrm{hr}^{-1}$ \\
\hline Synthesis of viral DNA & $\kappa$ & $7.11 \times 10^{-2}$ & $6.65 \times 10^{-2}$ & $\mathrm{hr}^{-1}$ \\
\hline Sequestration of viral DNA for encapsulation & $q$ & $2.08 \times 10^{-2}$ & $2.08 \times 10^{-2}$ & $\mathrm{hr}^{-1}$ \\
\hline Packaging and secretion of virions & $p$ & $5.36 \times 10^{-2}$ & $5.83 \times 10^{-2}$ & $\mathrm{hr}^{-1}$ \\
\hline Viral DNA per lytic host cell & $n_{T}$ & 69 & 68 & - \\
\hline Induced reactivation & $\alpha_{c}$ & $5.51 \times 10^{-1}$ & $1.40 \times 10^{-1}$ & $\mathrm{hr}^{-1}$ \\
\hline Induced death & $\delta_{c}$ & $5.13 \times 10^{-3}$ & $6.76 \times 10^{-3}$ & $\mathrm{hr}^{-1}$ \\
\hline
\end{tabular}


Table 2

Estimated parameter values, standard errors, and confidence intervals

\begin{tabular}{|c|c|c|c|c|}
\hline Data & Parameter & Estimated Value & Standard Error & Confidence Interval \\
\hline Zoeteweij, et al. & $\begin{array}{l}\alpha_{c} \\
\delta_{c}\end{array}$ & $\begin{array}{l}5.51 \times 10^{-1} \\
5.13 \times 10^{-3}\end{array}$ & $\begin{array}{l}1.03 \times 10^{-2} \\
3.25 \times 10^{-3}\end{array}$ & $\begin{array}{l}{\left[5.29 \times 10^{-1}, 5.73 \times 10^{-1}-1\right.} \\
{\left[-1.83 \times 10^{-3}, 1.21 \times 10^{-2}\right]}\end{array}$ \\
\hline $\mathrm{Yu}$, et al. & $\begin{array}{l}\alpha_{c} \\
\delta_{c}\end{array}$ & $\begin{array}{l}1.40 \times 10^{-1} \\
6.76 \times 10^{-3}\end{array}$ & $\begin{array}{l}7.84 \times 10^{-3} \\
2.88 \times 10^{-3}\end{array}$ & 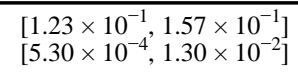 \\
\hline
\end{tabular}

